\title{
Root Patterning: Tuning SHORT ROOT Function Creates Diversity in Form
}

\author{
Marcela Hernández-Coronado and Carlos Ortiz-Ramírez* \\ UGA Laboratorio Nacional de Genómica para la Biodiversidad, CINVESTAV Irapuato, Guanajuato, Mexico
}

Roots have a fundamental role in plant growth and adaptation to different environments. Diversity in root morphology and architecture enables plants to acquire water and nutrients in contrasting substrate conditions, resist biotic and abiotic stress, and develop symbiotic associations. At its most fundamental level, morphology is determined by discrete changes in tissue patterning. Differences in the number and arrangement of the cell layers in the root can change tissue structure, as well as root length and girth, affecting important productivity traits. Therefore, understanding the molecular mechanisms controlling variation in developmental patterning is an important goal in biology. The ground tissue (GT) system is an ideal model to study the genetic

OPEN ACCESS

Edited by:

Raffaele Dello loio,

Sapienza University of Rome, Italy

Reviewed by:

Hongchang Cui,

Florida State University, United States Riccardo Di Mambro,

University of Pisa, Italy

*Correspondence:

Carlos Ortiz-Ramírez

carlos.ortiz@cinvestav.mx

Specialty section:

This article was submitted to Plant Development and EvoDevo, a section of the journal

Frontiers in Plant Science

Received: 22 July 2021 Accepted: 08 September 2021 Published: 30 September 2021

Citation:

Hernández-Coronado $M$ and

Ortiz-Ramírez C (2021) Root Patterning: Tuning SHORT ROOT Function Creates Diversity in Form.

Front. Plant Sci. 12:745861. doi: 10.3389/fp/s.2021.745861 basis of morphological diversity because it displays great interspecific variability in cell layer number. In addition, the genetic circuit controlling GT patterning in Arabidopsis thaliana has been well described, although little is known about species with more complex root anatomies. In this review, we will describe the Arabidopsis model for root radial patterning and present recent progress in elucidating the genetic circuitry controlling GT patterning in monocots and the legume Medicago truncatula (Mt), species that develop roots with more complex anatomies and multilayered cortex.

Keywords: root patterning, arabidopsis, short root, monocots, morphological diversity, root development, nodulation

\section{INTRODUCTION}

Root morphological diversity can be studied at different scales: macroscopically (i.e., at the level of root architecture, length, branching angle, and number of secondary roots) and microscopically, as changes in tissue pattering, like the number and arrangement of cell layers. Although root system architecture has been studied extensively, the relationship between patterning and root phenotypic diversity and adaptation has seldom been explored. Recently, it was proposed that root morphological trait variation across species and across biomes is most strongly influenced by root diameter, a character that depends on tissue layer number and organization (Ma et al., 2018).

Roots consist of three fundamental tissue types arranged radially as concentric layers: the epidermis on the outside; the ground tissue at the middle, consisting of one layer of endodermis and one to many layers of cortex; and a core of vascular elements plus 
pericycle called the stele (Dolan et al., 1993). Differences in the number and/or arrangement of these cell layers are not only relevant to explain diversity in root form, but they also give rise to important functional traits that have an impact on plant fitness and productivity (Lux et al., 2004). For example, plants living in waterlogged soils, such as rice, develop air filled cells from the cortex called aerenchyma (Yamauchi et al., 2018). Desert plants can accumulate water in their roots thanks to the proliferation of storage parenchyma from cortical cells, and a similar process allows plants to save starch to overwinter in difficult weather (Fahn, 1990; Lux and Inanaga, 1997). Halophyte plants growing in soils with toxic salt levels develop two suberized endodermis layers instead of one, which helps to isolate the root vasculature from the apoplast that is in direct contact with the soil (Inan et al., 2004).

At the basis of these developmental adaptations is the activity of genetic circuits that coordinate cell division activity and cell identity at the root apical meristem (RAM). Here, a set of stem cells, called initials, divide asymmetrically to produce daughter cells that give rise to epidermal, ground tissue, and vascular lineages (Esau, 1977; Benfey et al., 1993). While anticlinal divisions of initial cells will contribute to root length, periclinal divisions generate extra cell layers along the radial axis increasing girth. For example, in Arabidopsis a periclinal division of the cortex/endodermal initial gives rise to a single endodermis and a single cortex cell layer (Scheres et al., 1996). However, in species with roots that have multiple cortical layers the cortex/endodermal initial may undergo several divisions early in development producing thicker roots (Heimsch and Seago, 2008). There is limited information on the regulation of genetic circuits that produce different morphologies between closely related plant species, and even between roots from the same plant. Therefore, comparative intra- and interspecific developmental studies between roots with different morphologies are important for pinpointing the genetic basis of variation.

In this context, the ground tissue system is an ideal model to make such comparative studies since (1) it shows great variation in patterning, not only between closely related species, but also between root types from the same plant. e.g., rice roots can have between two to eleven ground tissue layers, depending on the root type (Clark and Harris, 1981). Maize develops between 8 and 15 layers (Bennetzen and Hake, 2009). (2) In many species, the ground tissue forms the bulk of the root; therefore, variations in the number of cortical cell layers have a significant effect on root form. (3) The genetic circuit controlling GT patterning in Arabidopsis is well described and serves as a model to search for modifications in the activity of homologous genes in species with more elaborated anatomies.

In this review, we will first describe the genetic circuit that controls development of the GT in Arabidopsis. Then, we will discuss the latest research in species with different anatomies and multilayered cortex. Finally, we will contrast the classical and new emergent models of root radial patterning and how they can be applied to explain morphological diversity.

\section{DIFFERENCES IN GT DEVELOPMENT}

Root tissue systems originate in the RAM, a region located near the tip of the root where cells remain undifferentiated. Here, a set of stem cell populations called initial cells undergo asymmetric cell division to produce different lineages. In Arabidopsis, there is four types of initials or stem cells: a population giving rise to vascular tissues, a population generating columella cells, a common initial producing the ground tissue (endodermis and cortex), and a common initial that originates root cap and epidermis (Pauluzzi et al., 2012). In the case of the ground tissue, the cortex/endodermis initials (CEIs) regenerate themselves by an anticlinal cell division, giving rise to the cortex endodermis initial daughter cell (CEID; Figures 1A,B). The CEID then undergoes one periclinal division to form two tissue layers: endodermis and cortex (Benfey et al., 1993). Thus, in primary development the Arabidopsis root has a single endodermal and a single cortex layer. Later in development an extra layer of cortex, called the middle cortex (MC), originates from the endodermis by an asymmetric periclinal division (Baum et al., 2002; Paquette and Benfey, 2005).

Important differences exist between root development in Arabidopsis and monocots, like rice and maize. In monocots, the epidermis and the ground tissue originate from the same initial cell. Therefore, a first asymmetrical division generates the CEI and the epidermis. Subsequently, the CEI will divide further giving rise to endodermis and cortex (Di Ruocco et al., 2018b; Figure 1B, left). Another important difference in the ability of most monocot roots to produce a multilayered cortex. It has been proposed that formation of several cortex layers is achieved by sustained periclinal cell divisions either of a single CEI or by divisions of multiple independent CEIs (Pauluzzi et al., 2012). In rice, there is a large variation in the number cortex cell layers depending on the root type. Small lateral roots have no cortex, while large laterals have 3; radical roots have 5, and crown roots have 10 (Clark and Harris, 1981). It is likely that precise modulation of the genetic circuit that controls CEI periclinal divisions underlies cortex layer number variation in rice, and this may be representative of a mechanism giving rise to interspecific ground tissue variation. Importantly, root diameter among rice root types is closely correlated with cortex cell layer number (Henry et al., 2015). Therefore, modulation of the CEI division program affects root size and function. Although this diversity in cortex development is most evident in monocots, it is not unique to this group. Some closely related dicot species also present differences in cortex layer number. For example, Cardamine hirsuta, a close Arabidopsis relative, has two cortex layers instead of one (Di Ruocco et al., 2018a). The wild tomato species Solanum pennellii has two cortex layers, while the domesticated Solanum lycopersicum has three, suggesting that differences in the genetic network controlling CEI divisions can evolve rapidly (Ron et al., 2013).

There is increasing evidence that the SHORT ROOT (SHR)/ SCARECROW (SCR) genetic pathway, initially described in Arabidopsis, also controls GT formation across species and that modifications in the activity of this pathway originate a multilayered cortex. 
A

Protein movement

High protein levels

Activation $\longrightarrow$ Repression
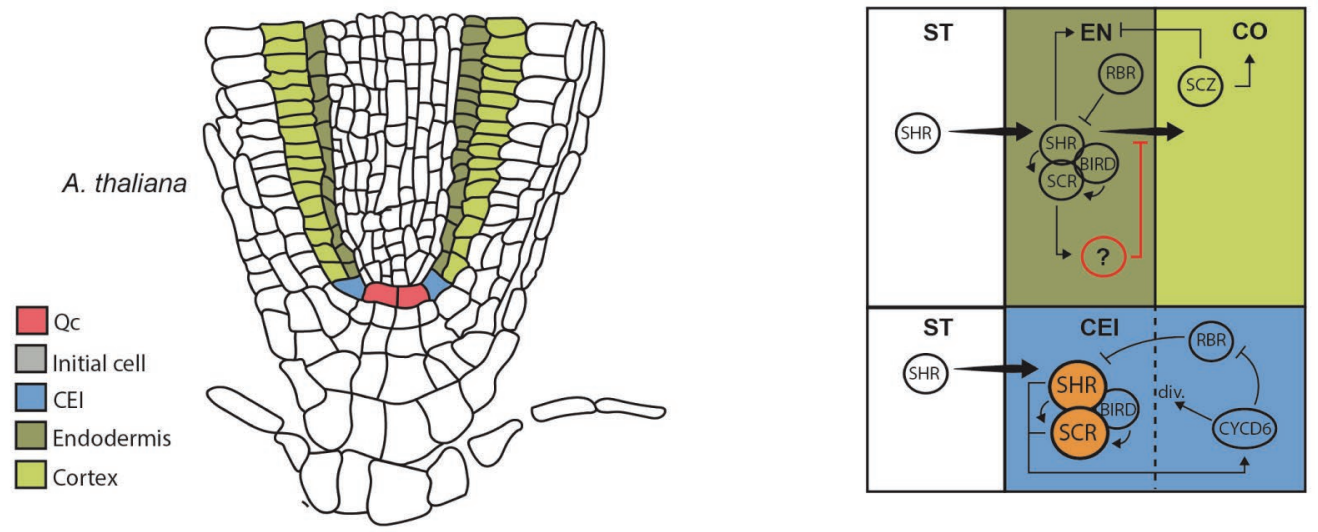

B
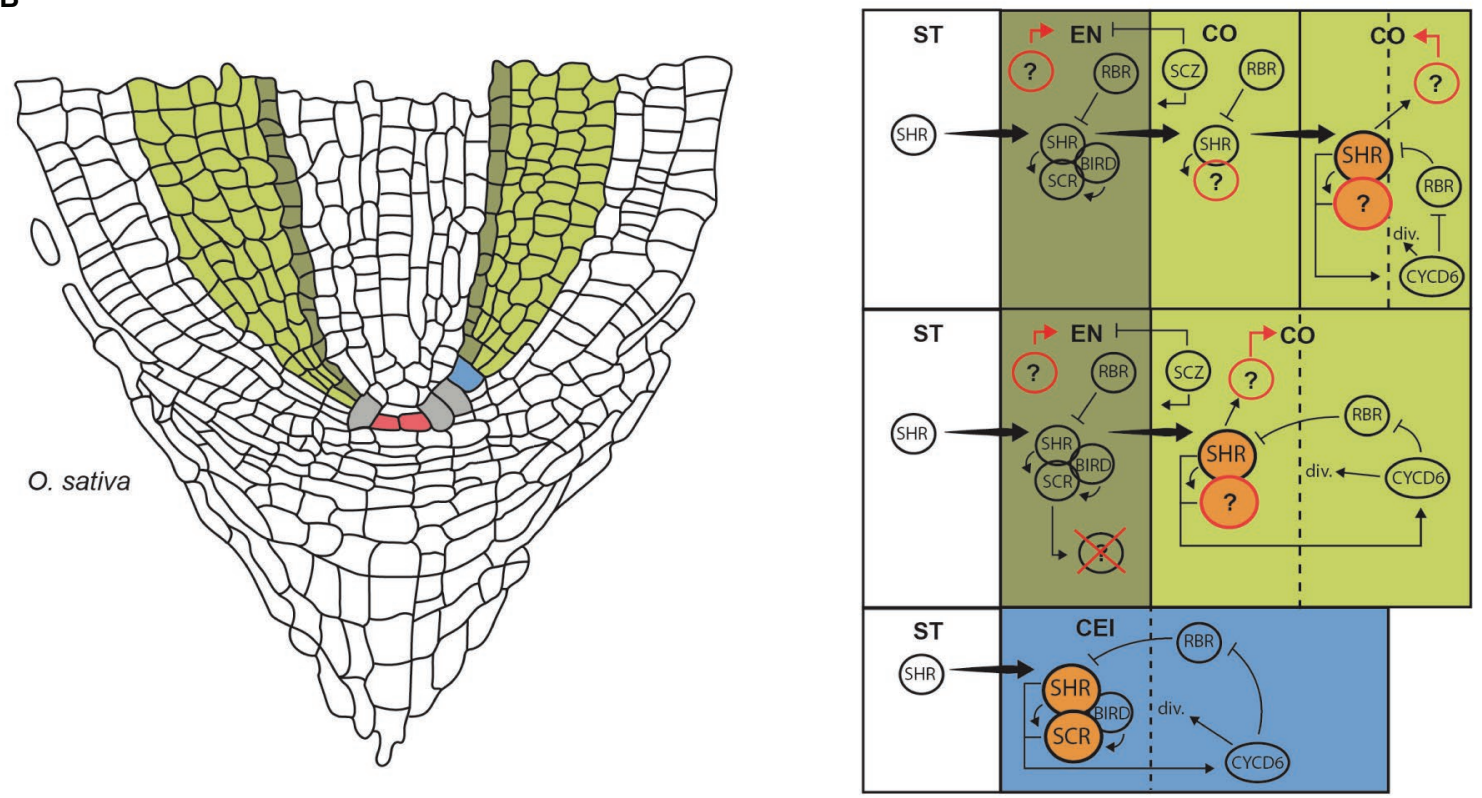

C
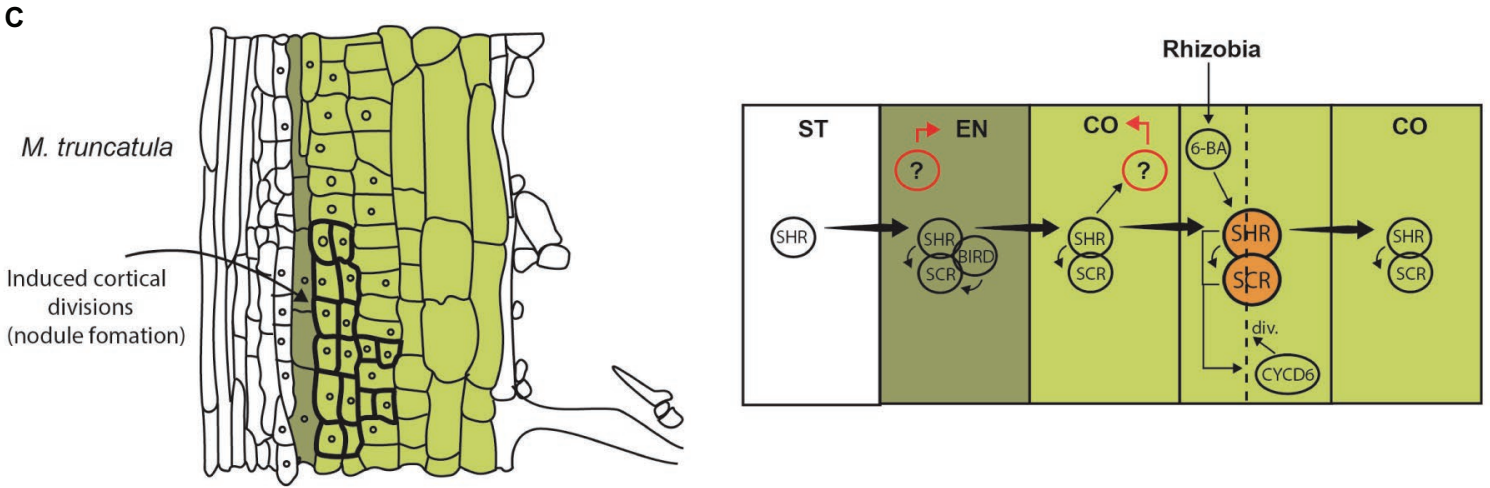

FIGURE 1 | Genetic models for GT formation and cortex expansion in different species. (A) Cartoon showing a longitudinal section from an Arabidopsis thaliana root highlighting initial and GT cells (left). Diagram representing the CEI, endodermis, cortex and stele, where the main genetic pathways contributing to GT formation are depicted (right). Briefly, SHR protein moves from the stele into the CEI where it induces SCR expression. SHR and SCR form a complex that moves 


\begin{abstract}
FIGURE 1 | into the nucleus and activates CYCD6;1, which in turns prevents the negative regulator RBR from interacting with the SHR/SCR complex. This results in high levels of SHR (big circles), which trigger asymmetric cell division giving rise to cortex and endodermis. Cell division resets the circuit from its on state. Presumably, SHR/SCR complex levels are maintained low by RBR preventing further divisions. SCZ helps in establishing tissue boundaries by inducing cortical identity and repressing endodermal identity. Importantly, SHR protein movement is limited to the endodermis either directly by biding to SCR and BIRD proteins, or indirectly by a SCR-induced factor that has been hypothesized to limit its movement (Wu et al., 2014; question mark). (B) Cartoon of a longitudinal sectional from rice root (left). Diagram representing the putative pathways contributing to GT formation and multilayered cortex in monocots (right). Note that SHR movement is not restricted to the endodermis; hence, the factor limiting its movement in Arabidopsis is either not present or has modified activity. We propose that SHR moves into the cortex where it can accumulate at high levels (big circles) activating and flipping the SHR/SCR/CYCLIND6;1/RBR circuit to its "on" state. This movement and activation are maintained until several cortical layers are produced. This model also assumes that SHR does not induce endodermal differentiation. Therefore, the factors that specify endodermal and cortical cell fate in monocots are unknown (question marks). It is also unclear if SHR interacts with SCR in the cortex as SCR protein localization in rice and other monocots is unknown. (C) Cartoon of a longitudinal sectional from Medicago root differentiation zone (left). Diagram representing the putative pathway contributing to cortex formative divisions giving rise to the nodule primordium (right). This model assumes that SHR and SCR proteins are present and interact throughout the root cortex. This allows the expression of the SHR/SCR/CYCLIND6;1/RBR circuit in cortical cells; however, the circuit is kept in an "off" state in the absence of rhizobia (low SHR levels). When symbiont signals are perceived by individual cells, 6-BA triggers local SHR accumulation in those domains flipping the circuit to its "on" state and triggering divisions.
\end{abstract}

\section{THE SHR/SCR PATHWAY CONTROLS GT FORMATIVE DIVISIONS}

As mentioned before, the asymmetric division of the CEI is essential for GT formation. Genetically, this process is controlled by the activity of $S H R$ and SCR transcription factors (TFs; Helariutta et al., 2000). These genes are members of the GRAS protein domain family, and together they activate the genetic pathway that determines radial patterning. Mechanistically, $S H R$ functions as a mobile signal that regulates cell division and cell fate specification. SHR transcript is expressed in the stele; however, the protein moves intercellularly through plasmodesmata into the adjacent CEI, CEID, and endodermis layer where it induces the expression of the downstream target SCR (Nakajima et al., 2001). SHR and SCR then form a complex at the protein level that moves into the nucleus of the CEI inducing the expression of a D-type cyclin, CYCLIND6;1 (CYCD6;1) in Arabidopsis. This triggers cell division allowing the differentiation of the clonally related cortex and endodermal cell layers (Sozzani et al., 2010; Figure 1A, right).

Importantly, this division of the CEI depends on a bistable circuit that functions as an on/off switch. Circuit stability is determined by CYCD6;1 activity and the cell cycle regulator RETINOBLASTOMA-RELATED protein $(R B R)$. Interaction of RBR with SCR reduces SHR/SCR complex formation, therefore decreasing SHR, SCR, and CYCD6;1 levels in a negative feedback loop that prevents cell division ("off" state; Cruz-Ramirez et al., 2012). At the same time, a positive feedback loop is encoded in this circuit because CYCD6;1 can negatively regulate RBR through phosphorylation (Figure 1A, right). Thus, increasing CYCD6; 1 activity shifts back the circuit to an "on" state. Simulations demonstrate that high levels of SHR influx into the CEI are needed to activate sufficient CYCD6;1 and gain the potential for asymmetric cell division. Moreover, very high levels ensure flipping to the "on" state triggering division. Finally, the bistable switch is reset when the cell undergoes division and proteins are degraded (Cruz-Ramirez et al., 2012).

Because SHR levels determine the potential of GT initial cells to undergo asymmetric divisions, the movement and magnitude of SHR fluxes into adjacent tissues have to be tightly regulated during GT formation. A prediction that can be extrapolated from current models is that further movement into the adjacent cell layers or GT initials will cause further activation of SCR and CYCD6;1 and thus cell division, increasing GT layer number. In Arabidopsis, it has been shown that SCR itself can regulate SHR movement by binding and sequestering SHR into the nucleus, which effectively limits the amount of protein that can move into the next layer (Heidstra et al., 2004; Cui et al., 2007). Therefore, SHR limits its own movement by inducing $S C R$ expression forming another feedback loop. This has been demonstrated by the reduction of SCR expression via RNAi, which results in increased SHR mobility and production of more GT layers (Cui et al., 2007). In addition, the SHR/ $S C R$ pathway induces the expression of the BIRD zinc finger proteins JACKDAW (JKD), MAGPIE (MGP), BALDIBIS, and NUTCRACKER that together with SCR also reduce SHR mobility and contribute to maintain tissue boundaries (Welch et al., 2007; Long et al., 2017).

After CEI division, the daughter cells acquire either endodermis or cortex identity. SHR also has an essential role in this process since experiments in Arabidopsis show it is necessary for endodermis cell fate specification. AtSHR loss-of-function roots lack an endodermis and display a single GT layer with cortex identity. On the other hand, SCR mutants also display a single GT layer, but this has a mix of endodermal and cortex markers. Importantly, double mutants of SCR and its close homolog SCARECROW-LIKE23 lack an endodermis similarly to SHR mutant, indicating that these TFs act together with $S H R$ to specify cell identity. (Benfey et al., 1993; van den Berg et al., 1995; Long et al., 2015). Another transcription factor that is important for defining GT cell fate during formative divisions is SCHIZORIZA (SCZ), a member of the heat-shock TF family. SCZ is important for cortex fate specification, since lossof-function mutants develop two ground tissue layers both with endodermis identity. Conversely, SCZ overexpression from the $35 \mathrm{~s}$ promoter results in ectopic cell divisions in epidermal and lateral root cap tissues in which high expression of the cortex marker Co2 is detected (ten Hove et al., 2010). Furthermore, SCZ has role in regulating correct cell fate separation and establishment of tissue boundaries across the root. Mutants show extra layers in ground, epidermal and lateral root cap tissues that express a mix of cell identity makers (Pernas et al., 2010). 


\section{MIDDLE CORTEX FORMATION}

In addition to activating cell division and specifying cell identity, SHR has a role in post-embryonic development of cortical tissue. In Arabidopsis roots, an additional cortex layer, called the middle cortex (MC), develops approximately 10-14 days after germination. This layer does not originate from the CEI as described previously, but from an asynchronous division of the endodermis that generates one extra cell layer that rapidly acquires cortex identity (Paquette and Benfey, 2005). SHR and PHABULOSA trigger this formative division through the reactivation of CYCD6; 1 (Sozzani et al., 2010; Bertolotti et al., 2021). However, the timing of this division is important and is regulated by the hormone gibberellic acid (GA). High levels of GA inhibit MC formation early after germination in a SHR-dependent process (Paquette and Benfey, 2005; Cui and Benfey, 2009). Surprisingly, SCR acts antagonistically to $S H R$ by repressing MC formation, as loss-of-function mutants initiate MC prematurely (Paquette and Benfey, 2005). Notably, the SHR, $S C R$, and GA pathways converge on the regulation of the transcription factor SCARECROW LIKE 3. This TF induces GA activity and itself is positively regulated by $S H R$ and $S C R$ forming a feedback loop that contributes to time MC formation (Heo et al., 2011; Koizumi et al., 2012; Gong et al., 2016).

\section{SHR IN THE DEVELOPMENT OF MULTILAYERED CORTEX}

In contrast to what has been reported in Arabidopsis, some functional aspects of SHR activity, such as mobility and fate specification, appear not to be conserved in other species. In recent years, compelling evidence has emerged for a role of $S H R$ in the specification and control of cortex layer number. Most of this new evidence comes from the study of SHR homologs in monocots, which develop roots with a multilayered cortex (Rebouillat et al., 2008). Notably, it was found that two SHR homologues from rice (Oryza sativa) and one homolog from Brachipodium distachyon have increased intercellular movement compared to Arabidopsis SHR. When expressed heterologously in the stele of Arabidopsis roots, movement of the monocot SHR proteins was not limited to the adjacent endodermis but instead they moved 3 to 6 layers past the stele. All monocot SHR proteins were shown to strongly interact with AtSCR, AtJKD, and AtMGP; hence, hypermobility could not be explained by a lack of interaction (and nuclear sequestration) between these transcription factors. The observed increase in SHR mobility was correlated with a similar increase in GT layer number. Therefore, SHR movement seems to be sufficient to activate the SHR/SCR/CYCLIND6;1 circuit causing extra divisions and an enlarged GT (Wu et al., 2014; Figure 1B, right). Importantly, by performing a propidium iodide exclusion test and analyses of cell-type specific markers, $\mathrm{Wu}$, et al. determined that the extra cell layers created by monocot SHR hypermobility had cortex identity. Hence, although monocot SHR homologs generate extra GT layers in Arabidopsis, just a single endodermis layer, the one in direct contact with the stele, was specified.

The fact that the experiments described before were performed in a heterologous system has both strengths and limitations. In this context, a recent study describing maize (Zea mays) endogenous $S H R$ function provides valuable insight into root development. Both ZmSHR transcript and protein localization differ from what has been reported in Arabidopsis. The transcript was detected not in the stele but in the root endodermis. This was corroborated by tissue-specific RNA expression data (FACSbased tissue isolation), single-cell RNAseq, and translational reporter lines (Ortiz-Ramirez et al., 2021). Although it is unclear if this expression pattern affects protein localization, translational reporter lines showed $Z m S H R$ protein is present in all cortical layers of the root (8-9 layers). This further confirms that SHR hypermobility is common in monocots. Moreover, generation of loss-of-function mutants in maize confirmed $Z m S H R$ role in multilayered cortex development. Roots harboring CRISPR mutant alleles for two ZmSHR homologs lacked several cortex layers but maintained a functional endodermis (Ortiz-Ramirez et al., 2021). Importantly, authors demonstrated that this function was conserved in monocots by generating CRISPR mutants in Setaria viridis. Similar to maize, double mutants displayed a strong reduction in cortex layer number (Ortiz-Ramirez et al., 2021). Conversely, an independent study revealed that overexpression of OsSHR2 in rice roots increases ground tissue layer number up to sixfold compared to wild type. Extra layers also had cortex identity (Henry et al., 2017).

In addition, there is evidence that SHR controls multilayer cortex formation in dicots as well. Cardamine hirsuta (Ch) is a close Arabidopsis relative that has been used as a model to study cortical expansion. Compared to Arabidopsis, Cardamine roots develop two cortex layers instead of one. SHR is also involved in cortex expansion in this species as heterologous expression of ChSHR in Arabidopsis produces roots with an additional cortex layer (Di Ruocco et al., 2018a). Therefore, a SHR-based mechanism to control cortex expansion seems to be conserved in many plant groups.

\section{SHR ENABLES NODULATION BY TRIGGERING CORTICAL CELL DIVISIONS}

Recently, SHR has been implicated in the development of root nodules, the structures that harbor the nitrogen fixing symbiont rhizobia. In Medicago truncatula, root nodule primordium is initiated upon rhizobia signals, which induce the dedifferentiation of cortical cells and its subsequent division (Timmers et al., 1999; Figure 1C, left). Both SHR and $S C R$ were found to be necessary for rhizobia-induced cortex cell divisions. As shown for monocot homologs, $M t S H R$ transcript and protein localization seem to be essential for cortex expansion. SHR expression in Medicago was found to be restricted to the stele, but the protein is present in the endodermis, cortex, and even the epidermis at low levels. Therefore, MtSHR intercellular movement is also expanded 
when compared to Arabidopsis. Notably, SCR protein was also observed in endodermis and cortex of Medicago roots (Dong et al., 2021).

Importantly, cortex-specific accumulation of SHR was found to be a key aspect of nodule formation. A decrease in nodule number was observed when MtSHR activity was specifically repressed in cortical cells using a SHR dominant repressor (MtSHR1-SRDX). In this line, inoculation with rhizobia did not trigger as many cell divisions as in wild type roots, which prevented formation of the nodule primordium. On the other hand, cortical cell-specific MtSHR overexpression induced divisions even in the absence of rhizobia (Dong et al., 2021). Thus, post-embryonic cortex expansion in Medicago is dependent on the local activation of the $S H R / S C R$ pathway. Importantly, the hormone cytokinin (6-BA) is produced in response to symbiont signals and seems to be upstream of $S H R$ in this local activation pathway. Authors showed that 6-BA triggered accumulation of $M t S H R-G U S$ and $M t S C R$, and promoted cortex divisions leading to nodule formation (Murray et al., 2007; Tirichine et al., 2007; Gauthier-Coles et al., 2018; Dong et al., 2021).

Moreover, Dong et al. (2021) demonstrated that not only the presence, but also the amount of SHR protein is crucial in triggering these divisions. As revealed by transgenic reporter lines, $M t S H R$ and $M t S C R$ proteins are present in the cortex prior rhizobia inoculation and activation of divisions. Hence, it is only when MtSHR is accumulated above a threshold level, either by symbiont-produced signals, hormones, or fusion to strong cortical-specific promoters (proMtNRT1.3), that cell division occurs (Figure 1C, right). Furthermore, it was found that rhizobia inoculation increases $M t S H R$ protein in cortical cells without modifying its transcript levels. This suggests that $M t S H R$ post-translational regulation in a cortex-specific context (e.g., by preventing protein degradation) represents a mechanism modulating cell division in Medicago roots.

\section{A REVISED MODEL OF ROOT RADIAL PATTERNING}

In the Arabidopsis-based model of root radial patterning, restriction of SHR movement through SCR interaction is a central regulatory aspect of the pathway that prevents development of more than one endodermal and one cortical cell layer. However, this function attributed to SCR do not seem to be conserved in other species. Both monocot and Medicago SCR homologs physically interact with SHR but do not limit its movement beyond the endodermis.

Alternatively, $\mathrm{Wu}$ et al. proposed that although $S C R$ role in driving SHR nuclear localization is conserved, the control of SHR mobility is not. Experiments show that nuclear sequestration alone cannot explain restriction of SHR movement; thus, another factor must be involved. For example, in Arabidopsis roots expressing monocot homologs, ectopic expression of AtSCR in the stele dramatically increases
OsSHR2 protein nuclear localization in this tissue. Because more protein is being held at the stele nuclei, a decrease in OsSHR2 intercellular movement should be observed. Nevertheless, no significant effect on the extent of protein mobility or decrease in cortex layer number was detected.

Another aspect of the model that requires further investigation is SHR role as an endodermal fate specification factor. In monocots and Medicago, the presence of SHR in the outer layers of the root does not result in the specification of endodermal identity. Rather, most of the GT layers where SHR protein is present have cortex identity according to their morphology and expression of genetic markers (Wu et al., 2014; Dong et al., 2021). Therefore, although SHR may be necessary, it is not sufficient to specify the root endodermis in these species.

A model for root radial patterning that incorporates the concept of SHR movement regulation as a central mechanism to control cortical layer number seems to be needed. In addition, work in Medicago suggest that local accumulation of SHR protein results in cell divisions and extra cortical layers, presumably by flipping the $S H R / S C R / C Y C D 6 ; 1 / R B R$ circuit to its "on" state. Therefore, SHR can be conceptualized as a cell division trigger which can be tuned by regulating protein movement, accumulation, and degradation in a cell-type specific context. This regulation in space and time ultimately sets the asymmetric cell division program that shapes root form and function.

\section{THE MISSING PIECES}

Although recent studies have enabled the identification of new regulatory aspects of the $S H R / S C R$ pathway, there are important questions regarding the identity of key regulatory components (see Figure 1 question marks). For example, the identification and characterization of the factors that modulate the extent of intercellular movement is paramount. Furthermore, SHR is important for the specification of endodermal identity in Arabidopsis, but this function is not conserved in monocots and Medicago. Thus, identifying the developmental genetic network that specifies endodermis and cortex cell fate in other species than Arabidopsis will contribute to elaborate a more complete model of root radial patterning. Finally, it is unclear if SHR and SCR protein domains overlap in species with multilayered cortex, in which SHR domain is expanded to all GT layers (Wu et al., 2014; Dong et al., 2021; Ortiz-Ramirez et al., 2021). In Medicago, SCR is expressed in endodermis and cortex; hence, both transcription factors have expanded domains that overlap (Dong et al., 2021). Further analyses in monocots to determine if this expansion is conversed in complex roots will be valuable.

To achieve this, comparative high-resolution gene expression analysis and development of transgenic reporter lines in species with contrasting GT patterning is needed. Single-cell RNAseq would enable detection of differences 
in gene activity at the level of individual cell layers, which is essential because many developmental regulators in the root act non-autonomously. In addition, generating highresolution gene expression networks for multiple species and comparing their architectures will contribute to map changes in genetic circuits underlying morphological diversity. Finally, SHR loss-of-function mutants in different species are needed. They will aid in dissecting SHR function in cortex development and proliferation, which is essential for harboring symbiotic associations, resist biotic and abiotic stress, and generating morphological diversity.

\section{REFERENCES}

Baum, S. F., Dubrovsky, J. G., and Rost, T. L. (2002). Apical organization and maturation of the cortex and vascular cylinder in Arabidopsis thaliana (Brassicaceae) roots. Am. J. Bot. 89, 908-920. doi: 10.3732/ajb.89.6.908

Benfey, P. N., Linstead, P. J., Roberts, K., Schiefelbein, J. W., Hauser, M. T., and Aeschbacher, R. A. (1993). Root development in Arabidopsis: four mutants with dramatically altered root morphogenesis. Development 119, 57-70. doi: $10.1242 /$ dev.119.1.57

Bennetzen, J.L., and Hake, S.C. (2009). Handbook of Maize: Its Biology. New York: Springer.

Bertolotti, G., Unterholzner, S. J., Scintu, D., Salvi, E., Svolacchia, N., Di Mambro, R., et al. (2021). A PHABULOSA-controlled genetic pathway regulates ground tissue patterning in the Arabidopsis root. Curr. Biol. 31, 420-426. doi: 10.1016/j.cub.2020.10.038

Clark, L. H., and Harris, W. H. (1981). Observations on the root anatomy of Rice (Oryza-Sativa-L). Am. J. Bot. 68, 154-161. doi: 10.1002/j.1537-2197.1981. tb12374.x

Cruz-Ramirez, A., Diaz-Trivino, S., Blilou, I., Grieneisen, V. A., Sozzani, R., Zamioudis, C., et al. (2012). A bistable circuit involving SCARECROWRETINOBLASTOMA integrates cues to inform asymmetric stem cell division. Cell 150, 1002-1015. doi: 10.1016/j.cell.2012.07.017

Cui, H., and Benfey, P. N. (2009). Interplay between SCARECROW, GA and LIKE HETEROCHROMATIN PROTEIN 1 in ground tissue patterning in the Arabidopsis root. Plant J. 58, 1016-1027. doi: 10.1111/j.1365-313X.2009.03839.x

Cui, H., Levesque, M. P., Vernoux, T., Jung, J. W., Paquette, A. J., Gallagher, K. L., et al. (2007). An evolutionarily conserved mechanism delimiting SHR movement defines a single layer of endodermis in plants. Science 316, 421-425. doi: 10.1126/science.1139531

Di Ruocco, G., Bertolotti, G., Pacifici, E., Polverari, L., Tsiantis, M., Sabatini, S., et al. (2018a). Differential spatial distribution of miR165/6 determines variability in plant root anatomy. Development 145:dev.153858. doi: 10.1242/ dev. 153858

Di Ruocco, G., Di Mambro, R., and Dello Ioio, R. (2018b). Building the differences: a case for the ground tissue patterning in plants. Proc. Biol. Sci. 285:20181746. doi: 10.1098/rspb.2018.1746

Dolan, L., Janmaat, K., Willemsen, V., Linstead, P., Poethig, S., Roberts, K., et al. (1993). Cellular organisation of the Arabidopsis thaliana root. Development 119, 71-84. doi: 10.1242/dev.119.1.71

Dong, W. T., Zhu, Y. Y., Chang, H. Z., Wang, C. H., Yang, J., Shi, J. C., et al. (2021). An SHR-SCR module specifies legume cortical cell fate to enable nodulation. Nature 589, 586-590. doi: 10.1038/s41586-020-3016-Z

Esau, K. (1977). Anatomy of Seed Plants. New York: John Wiley \& Sons.

Fahn, A. (1990). Plant Anatomy. 4th Edn. Oxford: Pergamon.

Gauthier-Coles, C., White, R. G., and Mathesius, U. (2018). Nodulating legumes are distinguished by a sensitivity to Cytokinin in the root cortex leading to Pseudonodule development. Front. Plant Sci. 9:1901. doi: 10.3389/fpls.2018.01901

Gong, X., Flores-Vergara, M. A., Hong, J. H., Chu, H., Lim, J., Franks, R. G., et al. (2016). SEUSS integrates gibberellin Signaling with transcriptional inputs from the SHR-SCR-SCL3 module to regulate middle cortex formation in the Arabidopsis root. Plant Physiol. 170, 1675-1683. doi: 10.1104/pp.15.01501

\section{AUTHOR CONTRIBUTIONS}

$\mathrm{MH}-\mathrm{C}$ and $\mathrm{CO}-\mathrm{R}$ wrote the article and designed the figures. All authors contributed to the article and approved the submitted version.

\section{FUNDING}

MH-C and CO-R are supported by UGA-LANGEBIO Cinvestav early career funds.

Heidstra, R., Welch, D., and Scheres, B. (2004). Mosaic analyses using marked activation and deletion clones dissect Arabidopsis SCARECROW action in asymmetric cell division. Genes Dev. 18, 1964-1969. doi: 10.1101/gad.305504

Heimsch, C., and Seago, J. L. Jr. (2008). Organization of the root apical meristem in angiosperms. Am. J. Bot. 95, 1-21. doi: 10.3732/ajb.95.1.1

Helariutta, Y., Fukaki, H., Wysocka-Diller, J., Nakajima, K., Jung, J., Sena, G., et al. (2000). The SHORT-ROOT gene controls radial patterning of the Arabidopsis root through radial signaling. Cell 101, 555-567. doi: 10.1016/ S0092-8674(00)80865-X

Henry, S., Dievart, A., Divol, F., Pauluzzi, G., Meynard, D., Swarup, R., et al. (2017). SHR overexpression induces the formation of supernumerary cell layers with cortex cell identity in rice. Dev. Biol. 425, 1-7. doi: 10.1016/j. ydbio.2017.03.001

Henry, S., Divol, F., Bettembourg, M., Bureau, C., Guiderdoni, E., Perin, C., et al. (2015). Immunoprofiling of Rice root cortex reveals two cortical subdomains. Front. Plant Sci. 6:1139. doi: 10.3389/fpls.2015.01139

Heo, J. O., Chang, K. S., Kim, I. A., Lee, M. H., Lee, S. A., Song, S. K., et al. (2011). Funneling of gibberellin signaling by the GRAS transcription regulator scarecrow-like 3 in the Arabidopsis root. Proc. National Acad. Sci. U. S. A 108, 2166-2171. doi: 10.1073/pnas.1012215108

Inan, G., Zhang, Q., Li, P., Wang, Z., Cao, Z., Zhang, H., et al. (2004). Salt cress. A halophyte and cryophyte Arabidopsis relative model system and its applicability to molecular genetic analyses of growth and development of extremophiles. Plant Physiol. 135, 1718-1737. doi: 10.1104/pp.104.041723

Koizumi, K., Hayashi, T., Wu, S., and Gallagher, K. L. (2012). The SHORTROOT protein acts as a mobile, dose-dependent signal in patterning the ground tissue. Proc. National Acad. Sci. U. S. A. 109, 13010-13015. doi: 10.1073/pnas.1205579109

Long, Y., Goedhart, J., Schneijderberg, M., Terpstra, I., Shimotohno, A., Bouchet, B. P., et al. (2015). SCARECROW-LIKE23 and SCARECROW jointly specify endodermal cell fate but distinctly control SHORT-ROOT movement. Plant J. Cell Mol. Biol. 84, 773-784. doi: 10.1111/tpj.13038

Long, Y., Stahl, Y., Weidtkamp-Peters, S., Postma, M., Zhou, W., Goedhart, J., et al. (2017). In vivo FRET-FLIM reveals cell-type-specific protein interactions in Arabidopsis roots. Nature 548, 97-102. doi: 10.1038/nature23317

Lux, A., and Inanaga, S. (1997). Die atypische Struktur der jungen Wurzeln einer Wüstensukkulente Echinocactus platyacanthus. Stapfia 50:5.

Lux, A., Luxová, M., Abe, J., and Morita, S. (2004). Root cortex: structural and functional variability and responses to environmental stress. Root Res. 13, 117-131. doi: 10.3117/rootres.13.117

Ma, Z., Guo, D., Xu, X., Lu, M., Bardgett, R. D., Eissenstat, D. M., et al. (2018). Evolutionary history resolves global organization of root functional traits. Nature 555, 94-97. doi: 10.1038/nature25783

Murray, J. D., Karas, B. J., Sato, S., Tabata, S., Amyot, L., and Szczyglowski, K. (2007). A cytokinin perception mutant colonized by rhizobium in the absence of nodule organogenesis. Science 315, 101-104. doi: 10.1126/ science. 1132514

Nakajima, K., Sena, G., Nawy, T., and Benfey, P. N. (2001). Intercellular movement of the putative transcription factor SHR in root patterning. Nature 413, 307-311. doi: 10.1038/35095061

Ortiz-Ramirez, C., Dias-Araujo, P. C., Zhang, C., Demesa-Arevalo, E., Yan, Z., $\mathrm{Xu}, \mathrm{X}$., et al. (2021). Ground tissue circuitry regulates organ complexity in cereal roots. BioRxiv. doi: 10.1101/2021.04.28.441892 
Paquette, A. J., and Benfey, P. N. (2005). Maturation of the ground tissue of the root is regulated by gibberellin and SCARECROW and requires SHORTROOT. Plant Physiol. 138, 636-640. doi: 10.1104/pp.104.058362

Pauluzzi, G., Divol, F., Puig, J., Guiderdoni, E., Dievart, A., and Perin, C. (2012). Surfing along the root ground tissue gene network. Dev. Biol. 365, 14-22. doi: 10.1016/j.ydbio.2012.02.007

Pernas, M., Ryan, E., and Dolan, L. (2010). SCHIZORIZA controls tissue system complexity in plants. Curr. Biol. 20, 818-823. doi: 10.1016/j.cub.2010.02.062

Rebouillat, J., Dievart, A., Verdeil, J. L., Escoute, J., Giese, G., Breitler, J. C., et al. (2008). Molecular genetics of Rice root development. Rice 2, 15-34. doi: 10.1007/s12284-008-9016-5

Ron, M., Dorrity, M. W., de Lucas, M., Toal, T., Hernandez, R. I., Little, S. A., et al. (2013). Identification of novel loci regulating interspecific variation in root morphology and cellular development in tomato. Plant Physiol. 162, 755-768. doi: 10.1104/pp.113.217802

Scheres, B., McKhann, H. I., and Van den Berg, C. (1996). Roots redefined: anatomical and Cenetic analysis of root development. Plant Physiol. 111, 959-964. doi: 10.1104/pp.111.4.959

Sozzani, R., Cui, H., Moreno-Risueno, M. A., Busch, W., Van Norman, J. M., Vernoux, T., et al. (2010). Spatiotemporal regulation of cell-cycle genes by SHORTROOT links patterning and growth. Nature 466, 128-132. doi: 10.1038/ nature 09143

ten Hove, C. A., Willemsen, V., de Vries, W. J., van Dijken, A., Scheres, B., and Heidstra, R. (2010). SCHIZORIZA encodes a nuclear factor regulating asymmetry of stem cell divisions in the Arabidopsis root. Curr. Biol. 20, 452-457. doi: 10.1016/j.cub.2010.01.018

Timmers, A. C., Auriac, M. C., and Truchet, G. (1999). Refined analysis of early symbiotic steps of the rhizobium-Medicago interaction in relationship with microtubular cytoskeleton rearrangements. Development 126, 3617-3628. doi: $10.1242 /$ dev.126.16.3617

Tirichine, L., Sandal, N., Madsen, L. H., Radutoiu, S., Albrektsen, A. S., Sato, S., et al. (2007). A gain-of-function mutation in a cytokinin receptor triggers spontaneous root nodule organogenesis. Science 315, 104-107. doi: 10.1126/ science. 1132397 van den Berg, C., Willemsen, V., Hage, W., Weisbeek, P., and Scheres, B. (1995). Cell fate in the Arabidopsis root meristem determined by directional signalling. Nature 378, 62-65. doi: 10.1038/378062a0

Welch, D., Hassan, H., Blilou, I., Immink, R., Heidstra, R., and Scheres, B. (2007). Arabidopsis JACKDAW and MAGPIE zinc finger proteins delimit asymmetric cell division and stabilize tissue boundaries by restricting SHORT-ROOT action. Genes Dev. 21, 2196-2204. doi: 10.1101/gad. 440307

Wu, S., Lee, C.-M., Hayashi, T., Price, S., Divol, F., Henry, S., et al. (2014). A plausible mechanism, based upon SHORT-ROOT movement, for regulating the number of cortex cell layers in roots. Proc. National Acad. Sci. U. S. A. 111, 16184-16189. doi: 10.1073/pnas.1407371111

Yamauchi, T., Colmer, T. D., Pedersen, O., and Nakazono, M. (2018). Regulation of root traits for internal aeration and tolerance to soil waterlogging-flooding stress. Plant Physiol. 176, 1118-1130. doi: 10.1104/ pp. 17.01157

Conflict of Interest: The authors declare that the research was conducted in the absence of any commercial or financial relationships that could be construed as a potential conflict of interest.

Publisher's Note: All claims expressed in this article are solely those of the authors and do not necessarily represent those of their affiliated organizations, or those of the publisher, the editors and the reviewers. Any product that may be evaluated in this article, or claim that may be made by its manufacturer, is not guaranteed or endorsed by the publisher.

Copyright (c) 2021 Hernández-Coronado and Ortiz-Ramírez. This is an open-access article distributed under the terms of the Creative Commons Attribution License (CC BY). The use, distribution or reproduction in other forums is permitted, provided the original author(s) and the copyright owner(s) are credited and that the original publication in this journal is cited, in accordance with accepted academic practice. No use, distribution or reproduction is permitted which does not comply with these terms. 